\title{
Place second-order stereological tool in the toolbox of the quantitative research
}

Keywords: histology, physiology, stereology

\section{Mini review}

Conventional stereological methods have been applied widely to estimate the quantitative aspects of the histological particles under different physiological or pathological conditions. ${ }^{1}$ These parameters are the volume, surface area, length, and number of the particles. However, important information also can be obtained in regards of the three-dimensional arrangement of the histological particles which are related directly to its function. ${ }^{1}$ It can be illustrated by remembrance of the neuronal arrangement and proximity of the glial cells to them. It has been documented that the neuron-neuron or neuron-glia arrangements are related directly to their proper interactions. ${ }^{2}$ Glial cells are fundamental functional components of the synapses and signaling and also take parts in nursing and feeding of the neurons. ${ }^{2}$ Although the second-order stereology can also provide supplementary information about the spatial arrangement of the components, it has received less attention. A variety of techniques has been presented to display the spatial arrangements of the cells or even organelles by researchers. Estimation of covariance " $\mathrm{C}(\mathrm{r})$ " and pair-correlation functions " $\mathrm{g}(\mathrm{r})$ " is one of these techniques to explore the threedimensional arrangements of cell-cell or organelle-organelle. This method has been applied in estimation of the spatial allocation of a variety of histological particles including histological components of the benign and malignant prostatic tissues, microstructures of the diabetic heart, the A-cell and B-cells of dorsal root ganglion, the neuron-glia of the frontal cortex of the stressed rats, and the sub cellular localization of the organelles in the acinar cells of salivary gland. ${ }^{3-7}$ Here, the $\mathrm{C}(\mathrm{r})$ and $\mathrm{g}(\mathrm{r})$ have illustrated spatial arrangement of the particles in the histological tissue samples. To use this technique, the covariance of a particle at a distance of " $\mathrm{r}$ " units into the histological reference space could be obtained using the "dipole". The dipoles are the number of lines with defined lengths. C(r) and $\mathrm{g}(\mathrm{r})$ could be estimated by counting linear dipole probes of different sizes superimposed on the histological sections. After obtaining the $\mathrm{C}(\mathrm{r})$ and $\mathrm{g}(\mathrm{r})$ the outcomes would be plotted. The plots explain how much spatial proximity of two particles might be changed. In another word these plots show how much the center of a particle are far from the center of another particle i.e. the covariance is related to how the particles are packed or dispersed. Once the particles demonstrate a more dispersed pattern, the probability of finding a couple of particle hitting with a larger dipole length would be increased. In case of two different particles, the term "cross-correlation function" is used. The term shows how different particles are packed or detached together. For example it can show clustering or dispersing of the microvessels and cardiomyocytes in the diabetic heart. ${ }^{4}$ To end this mini review, it is suggested that to place the second-order stereological methods in the toolbox of the quantitative research, since it can illuminate some of the shady edge of the histological and physiological research.
Volume 5 Issue I - 2018

\author{
Ali Noorafshan ${ }^{1,2}$ \\ 'Histomorphometry and Stereology Research Center, Shiraz \\ University of Medical Sciences, Iran \\ ${ }^{2}$ Department of Anatomy, Shiraz University of Medical Sciences, \\ Iran \\ Correspondence: Ali Noorafshan, Histomorphometry and \\ Stereology Research Center, Shiraz University of Medical \\ Sciences, Shiraz, Iran, Tel: +987| 32304372 , \\ Email noora@sums.ac.ir
}

Received: July 24, 2016 | Published: January 10, 2018

\section{Support}

The manuscript was supported by Histomorphometry and Stereology Research Center, Shiraz University of Medical Sciences, Shiraz, IR Iran.

\section{Acknowledgements}

None.

\section{Conflict of interest}

Author declares that there is no conflict of interest.

\section{References}

1. Reed MG, Howard CV, De Yanes GS. One-stop stereology: the estimation of 3D parameters using isotropic rulers. J Microsc. 2010;239(1):54-65.

2. Araque A, Navarrete M. Glial cells in neuronal network function. Philos Trans R Soc Lond B Biol Sci. 2010;365(1551):2375-2381.

3. Mattfeldt T, Eckel S, Fleischer F, et al. Statistical analysis of reduced pair correlation functions of capillaries in the prostate gland. $J$ Microsc. 2006;223(Pt 2):107-119.

4. Noorafshan A, Karbalay Doust S, Khazraei H, et al. Spatial arrangement of the heart structure: Application of second-order stereology in diabetic rats. Ann Anat. 2014;196(1):20-25.

5. Noorafshan A, Omidi A. Spatial arrangements of dorsal root gan $\neg$ glion cells after sciatic nerve crush. Micron. 2011;42(8):905-910.

6. Noorafshan A, Abdollahifar MA, Karbalay Doust S. Stress changes the spatial arrangement of neurons and glial cells of medial prefrontal cortex and sertraline and curcumin prevent it. Psychiatry Investig. 2015;12(1):73-80.

7. Mayhew TM. Quantitative description of the spatial arrangement of organelles in a polarised secretory epithelial cell: the salivary gland acinar cell. J Anat. 1999;194(Pt 2):279-285. 